\title{
Urabstimmung FMH: Stimmen wir JA!
}

Die Gesetzesvorlage 04.062 Managed Care hat ein entscheidendes Problem: Niemand braucht sie! - Ärztinnen und Ärzten in Netzwerken nützt sie nichts. Medizinerinnen und Mediziner ausserhalb eines gemanagten Netzwerkes brauchen sie nicht. In der Schweiz sind bereits viele (Haus-) Ärztinnen und Ärzte in verschiedenen Organisationen und Netzwerken zusammengeschlossen. Diese Entwicklung soll auch in Zukunft freiheitlich und freiwillig erfolgen.

Rund 25000 der 35000 FMH-Mitglieder rufen die Schweizer Ärzteschaft auf, sich gegen die Vorlage 04.062 zu stellen: vier Kantonalgesellschaften (BL, BS, GE, SH), drei Fachgesellschaften (Dermatologen, Ophthalmologen, ORL), der VSAO und Psica.ch - Wichtig: Diese Gesellschaften sind nicht grundsätzlich gegen Managed Care, bekämpfen jedoch die aktuelle Vorlage 04.062 und den Zwang zu diesem System!

\section{Warum sind diese Gesellschaften gegen die Vorlage 04.062?}

Weil sie die freie Arztwahl abschafft, für Netzwerke Budgetmitverantwortung obligatorisch macht, den Kontrahierungszwang aushöhlt und das Patientengeheimnis schwächt.

\section{Gründe für ein JA bei der Urabstimmung:}

- Wir sind die wichtigsten Fürsprecher unserer Patienten und auch verantwortlich für die Zukunft der Ärzteschaft.

- Das bestehende System mit einem gesunden Mix aus gemanagten Netzwerken und informellen Netzen aus Gruppen- und Einzelpraxen ist austariert und hat sich bestens bewährt.

- Die Managed-Care-Vorlage 04.062 plant einen Radikalumbau des etablierten, qualitativ hochstehenden Schweizer Gesundheitswesens.

- Die Managed-Care-Vorlage 04.062 mit der Abschaffung der freien Arztwahl schadet sowohl uns Ärzten als auch unseren Patienten.

- Die bisherige FMH-Politik mit enger Anlehnung an «Bundesbern» hat uns vor gravierenden Fehlentwicklungen nicht verschont.

- Durch die FMH-Politik der übermässigen Kompromissbereitschaft werden zentrale Grundwerte der ärztlichen Tätigkeit aufgegeben.

- Sämtliche in den Kantonal- und Fachgesellschaften durchgeführten Befragungen und Abstimmungen haben eine fast einstimmige Ablehnung eines Zwangs zu Managed Care ergeben.

- Wir brauchen vor keinem Drohszenario Angst zu haben. Eine geeinte FMH vermag durch ihre 35000 Mitglieder mit gezielten Aktionen genügend Stimmbürger zu mobilisieren, um einen massgebenden Einfluss auf politische Entscheidungen zu nehmen.

\section{Standespolitische Überlegungen}

«Wer A sagt, muss nicht unbedingt B sagen. Er kann auch erkennen, dass A falsch war.» (Bertold Brecht)

Es trifft zu, dass die Ärzte selbst einige Eckpfeiler der Vorlage 04.062 vorgeschlagen haben. Das Thesenpapier «Ja zu Managed Care» wurde im Dezember 2006 von der Ärztekammer jedoch unter der Prämisse angenommen, dass die Budgetmitverantwortung freiwillig sein solle.

Wenn eine Ärztin oder ein Arzt freiwillig in einem Netzwerk mit Budgetmitverantwortung arbeiten will und die Versicherten/Patienten über die Budgetmitverantwortung der Ärzte informiert sind, hat niemand etwas dagegen. Diese Art von Netzwerken gibt es schon heute.

Es darf aber keine Ärztin/kein Arzt und kein Patient in ein solches Modell gezwungen werden. Genau dies sieht aber die Vorlage 04.062 vor.

\section{Jetzt ist der richtige Zeitpunkt für einen Referendumsbeschluss}

Die Eckpfeiler der Vorlage (zwingende Budgetmitverantwortung für Netzwerke, differenzierter Selbstbehalt) können vom Parlament nun nicht mehr geändert werden.

Wegen dieser für uns inakzeptablen Inhalte der Gesetzesvorlage braucht es jetzt ein klares Signal an das Parlament, damit die Vorlage beerdigt wird.

Es ist bereits jetzt bekannt, dass nach Verabschiedung von 04.062 das Referendum dagegen ergriffen wird. Verschiedene Gruppierungen haben dies bereits angemeldet.

Die zwingende Budgetmitverantwortung ist eine zu einschneidende Massnahme, als dass wir Ärztinnen und Ärzte sie akzeptieren könnten! Die FMH sollte daher bei einem Referendum nicht abseits stehen und möglichst viele Stimmbürger gegen die Vorlage 04.062 mobilisieren.

\section{Die Vorlage 04.062 muss als Ganzes abgelehnt werden}

Ja, die Vorlage 04.062 ist so schlecht, dass es keinen Sinn macht, sie noch weiter verbessern zu wollen. Anliegen, welche die Ärzteschaft noch einbringen will wie die Forderung, dass Netzwerke unabhängig sein sollen (Motion Cassis) - sind zwar lobenswert, beseitigen die inakzeptablen Inhalte von 04.062 jedoch nicht. 


\section{Verteidigung unserer Grundwerte anstatt «Taktik»}

Noch vor drei Jahren hat die FMH mit Erfolg für den Erhalt der freien Arztwahl gekämpft (Eidgenössische Abstimmung vom 1. Juni 2008). Nun, im Jahr 2011, soll die freie Arztwahl mit der Vorlage 04.062 abgeschafft werden, und die FMH-Führung erklärt sich damit einverstanden.

Als Grund dafür wird «Taktik» angeführt. Man wolle verhindern, dass sonst der Kontrahierungszwang aufgelöst werde. Dieses Drohszenario ist ein altbekannter Trick. Man gibt vor, dass zwischen zwei Übeln gewählt werden müsse. Dabei wäre die FMH stark, wenn sie geeint auftreten würde, mit der Legitimation der Basis im Rücken.

«Taktik» gehört in die Welt der Politiker. Als Ärztinnen und Ärzte (35000 FMH-Mitglieder) können wir in der demokratischen Schweiz Einfluss auf politische Entscheidungen nehmen, indem wir uns mit unseren Kernanliegen aktiv einbringen. Wir sollten uns nicht auf eine falsch verstandene Kompromissbereitschaft einlassen. Gewisse Grundwerte (z. B. Schutz der vertrauensvollen Arzt-Patienten-Beziehung) sind nicht verhandelbar.

\section{Die Vorlage 04.062 ist unnötig und hat lediglich die Rationierung von KVG-Leistungen zum Ziel}

Die Vorlage 04.062 ist kein Rezept gegen den demographisch bedingten Kostenanstieg im Gesundheitswesen.

Ärztinnen und Ärzte arbeiten bereits heute vernetzt und kostenbewusst nach WZW-Kriterien, sei es in einem der bestehenden «Netzwerke» oder im informellen Netz. Die Vorlage 04.062 ist deshalb unnötig! Mit dem differenzierten Selbstbehalt sollen Patienten und Ärzte dazu bewegt werden, sich einem offiziellen Netzwerk anzuschliessen. Gleichzeitig wird in den Netzwerken mit der zwingenden Budgetmitverantwortung die Schraube von den Versicherern angezogen.

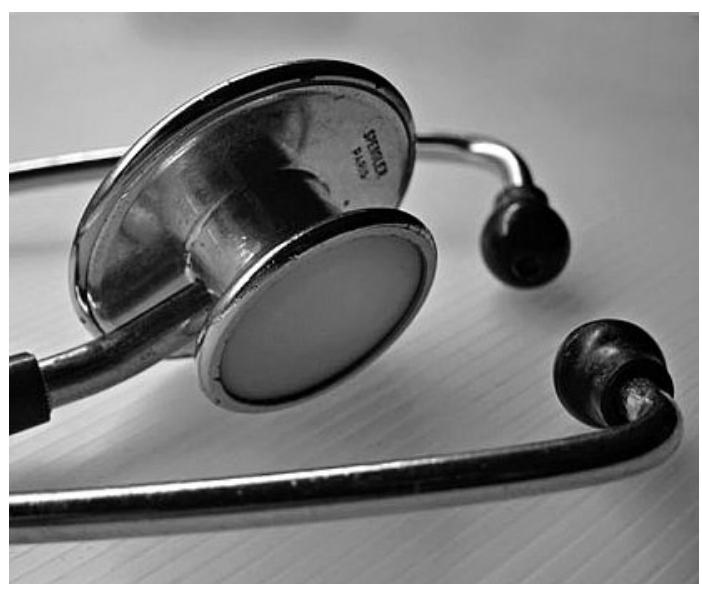

Ärztinnen und Ärzte werden immer mehr unter Druck geraten, die Budgetvorgaben werden je länger je strenger werden. Dies mündet in eine Rationierung der Medizin. Anstatt dass die Politik und die Bevölkerung diskutieren, ob und wenn ja welche Rationierungen bei steigendem Kostendruck vorgenommen werden dürfen, wird der schwarze Peter den Ärzten zugeschoben.

Die medizinische und die ökonomische Verantwortung sind heute in der Regel getrennt (behandelnder Arzt versus Vertrauensarzt). Wenn diese Trennung wegfällt und die fachlich-medizinischen Entscheidungen massgebend von der ökonomischen Verantwortung beeinflusst werden, zerstört dies das Vertrauensverhältnis zwischen Arzt und Patient.

\section{Für eine geeinte Ärzteschaft}

Mit der Vorlage 04.062 werden wir Ärztinnen und Ärzte gegeneinander ausgespielt. Es wird behauptet, dass die medizinische Qualität in offiziellen Netzwerken besser sei als ausserhalb. Dies ist eine Annahme, die nicht bewiesen ist, genauso wenig wie die Behauptung, gemanagte Netzwerke würden kostengünstiger arbeiten.

Neun Gesellschaften mit 25000 Mitgliedern fordern, dass sich die FMH konsequent gegen die vom Parlament geplante Managed-Care-Vorlage 04.062 und die damit verbundene Abschaffung der freien Arztwahl (durch den differenzierten Selbstbehalt), gegen die zwingende Budgetmitverantwortung für Netzwerke, gegen die Vertragsfreiheit für Netzwerke und gegen die Aushöhlung des Patientengeheimnisses einsetzt. Die FMH soll aktiv und nachweislich für diese Ziele einstehen, was auch die Unterstützung eines allfälligen Referendums gegen eine derart gestaltete Managed-Care-Vorlage beinhaltet.

Ärztegesellschaft Baselland, Medizinische Gesellschaft Basel, Association des Médecins du Canton de Genève, Kantonale Ärztegesellschaft Schaffhausen, Société Suisse de Dermatologie, Société Suisse d'Ophtalmologie, Société Suisse d'ORL, www.psica.ch

\section{JA zu meinem Beruf:}

- Meiner Freiheit

- Meiner Unabhängigkeit

- Meiner Zukunft

JA zu meinen Patienten:

- Ihrer Freiheit

- Ihrer Gesundheit

- Ihrer Wahl

Die Entscheidung liegt bei uns! - Stimmen wir JA! 


\section{Ja zum Urabstimmungsantrag}

\begin{abstract}
Der VSAO begrüsst grundsätzlich die integrierte Gesundheitsversorgung. Besonders für chronisch kranke Menschen ist es wesentlich, dass sich Versorgungsnetzwerke bilden, in denen die verschiedenen Leistungserbringer (Hausärztinnen und -ärzte, Spitex, Spezialpraxen, Kliniken usw.) optimal zusammenarbeiten. Damit wird die Qualität der Versorgung verbessert, und teure Doppelspurigkeiten können vermieden werden. Die Managed-Care-Vorlage läuft aber nach Ansicht des VSAO in eine falsche Richtung.
\end{abstract}

\section{Warum jetzt?}

Mit der Referendumsdrohung senden die FMHMitglieder den National- und Ständerätinnen und -räten ein deutliches Signal im Hinblick auf die Schlussabstimmung im Parlament vom 30. September.

Es ist Zeit, dass die Ärztinnen und Ärzte ein klares Zeichen gegen die schlecht durchdachte ManagedCare-Vorlage setzen.

Für den VSAO stehen dabei folgende Argumente im Vordergrund:

\section{Abschaffung der freien Arztwahl}

Das Parlament plant derzeit, für 2012 diejenigen Patienten, die sich nicht in einem integrierten Versorgungsmodell versichern wollen, finanziell empfindlich zu bestrafen, insbesondere durch sogenannte «differenzierte» Selbstbehalte. Weil all jene Versicherten, die sich nicht einem Netzwerk anschliessen, mit einem doppelt so hohen Selbstbehalt belastet werden sollen, müsste die «freie» Arztwahl künftig teuer erkauft werden. Viele Patienten würden praktisch gezwungen, einem ManagedCare-Modell beizutreten.

\section{Die Managed-Care-Vorlage läuft nach Ansicht des VSAO in eine falsche Richtung.}

\section{Problem Budgetmitverantwortung}

Integrierte Versorgungsnetze müssen gemäss Vorlage zwingend Budgetmitverantwortung tragen. Was als Mitverantwortung beginnt, wird im Rahmen der Sparbemühungen rasch zur vollen Budgetverantwortung werden.
Der VSAO warnt aus folgenden Gründen bereits seit Jahren vor der Budgetverantwortung:

- Sie führt zu einer verdeckten Rationierung und zu einem unvermeidbaren Qualitätsverlust.

- Es entsteht ein Anreiz zur Unterversorgung.

- Leistungserbringer werden zur Risikoselektion gedrängt.

- Das Vertrauensverhältnis zwischen Arzt und Patient wird gestört, weil medizinische und ökonomische Verantwortung nicht mehr getrennt sind.

\section{Problem Vertragsfreiheit}

Nach dem Willen des Parlaments sollen die Krankenkassen zudem eigenmächtig bestimmen können, mit welchen (kostengünstigen) Versorgungsnetzwerken sie Verträge abschliessen wollen und mit welchen nicht. Der Kontrahierungszwang würde somit ausgehöhlt.

Es ist Zeit, dass die Ärztinnen und Ärzte ein klares Zeichen gegen die schlecht durchdachte ManagedCare-Vorlage setzen.

\section{Problem Patientengeheimnis}

Dazu kommt, dass der zur «Optimierung» der Behandlungen geforderte Datenaustausch innerhalb der Netzwerke - Stichwort «gläserner Patient» - das Patientengeheimnis, also die vertrauensvolle ArztPatienten-Beziehung, empfindlich schwächt. Gerade bei der Behandlung von stigmatisierten, z. B. psychischen Erkrankungen, kann der jetzt schon praktizierte Datentransfer in Netzwerken unheilvolle Auswirkungen auf die Arzt-Patienten-Beziehung haben.

\section{Fazit}

Diese gesundheitspolitischen Pläne gefährden unsere im weltweiten Vergleich hervorragende Gesundheitsversorgung. Die Abschaffung der in der Schweiz bewährten freien Arztwahl darf nicht vom Parlament im Alleingang entschieden werden. Vor einem so tiefgreifenden und unwiderruflichen Systemwechsel im Schweizer Gesundheitswesen muss das Stimmvolk befragt werden. 